\title{
Um estudo longitudinal de tempo e aspecto na demência do tipo Alzheimer
}

\author{
A longitudinal study of tense and aspect in the dementia of the Alzheimer type
}

\author{
Juliana Barros Nespoli \\ Celso Vieira Novaes \\ Universidade Federal do Rio de Janeiro - Rio de Janeiro - Brasil
}

\begin{abstract}
Resumo: O objetivo deste estudo é investigar possíveis alterações na expressão linguística de tempo e aspecto de pacientes portadores da demência do tipo Alzheimer (DTA). Principalmente, objetiva-se contribuir para o entendimento acerca do comprometimento cognitivo subjacente a essas eventuais alterações. Para tanto, foi desenvolvido um estudo de caso de caráter longitudinal. Foi selecionado um paciente portador da DTA, que foi submetido a quatro aplicações de duas avaliações de naturezas distintas: uma avaliação neuropsicológica e uma avaliação linguística. Foram detectadas alterações na expressão linguística de tempo e aspecto. A análise desses resultados sugere que as alterações detectadas até a terceira aplicação podem decorrer de um comprometimento no sistema linguístico ou em sistemas não linguísticos. Já o declínio na expressão linguística de tempo e aspecto detectado na quarta aplicação sugere um comprometimento no sistema linguístico.
\end{abstract}

$\diamond$

Palavras-chave: Tempo; Aspecto; DTA; Estudo longitudinal

\begin{abstract}
This study aims to investigate any possible impairment related to the linguistic expression of tense and aspect by patients with the dementia of the Alzheimer type (DAT). Mainly, it aims to contribute to the understanding of the cognitive disruption underlying these eventual impairments. In order to achieve these aims, it was developed a case study of longitudinal nature. It was selected a DAT patient, who was submitted four times to two different evaluations: a neuropsychological evaluation and a linguistic evaluation. Impairments in the linguistic expression of tense and aspect were detected. The analysis of the results suggests that the impairments detected up to the third assessment may have been originated due to a disruption of the linguistic system or of non-linguistic systems. The decline in the linguistic expression of tense and aspect detected in the fourth assessment suggests a disruption of the linguistic system.
\end{abstract}

Keywords: Tense; Aspect; DAT; Longitudinal study

\section{Introdução}

Os diferentes estudos acerca da linguagem podem ser norteados por perspectivas e propósitos distintos. O Gerativismo, teoria linguística adotada neste trabalho, tem como um de seus objetivos a investigação da representação mental da linguagem. Podemos traçar considerações a respeito da representação dos fenômenos linguísticos tanto a partir da investigação da expressão linguística de indivíduos adultos saudáveis quanto a partir da avaliação da expressão linguística de indivíduos cuja gramática mental pode ser caracterizada como desviante ${ }^{1}$. Dessa maneira, proposições teóricas podem ser validadas ou não por meio da avaliação da expressão linguística de diferentes populações.

No que se refere à avaliação da expressão linguística de indivíduos que apresentam uma gramática desviante, podemos dizer que os desvios encontrados permitem que sejam traçadas considerações teóricas. Por exemplo, ao analisar dados provenientes dessa população, podemos constatar a presença de uma determinada propriedade

\footnotetext{
1 Considera-se desviante a gramática cujas propriedades diferem daquela de um indivíduo adulto e saudável.
} 
linguística $\mathrm{X}$ em meio à ausência de uma propriedade linguística $\mathrm{Y}$. Tal constatação pode ser um indicativo de que $\mathrm{X}$ e Y são propriedades linguísticas dissociadas na gramática não desviante, o que pode ser revelador para uma teoria que busca o entendimento da representação mental da linguagem.

Um exemplo de gramática desviante é a gramática de uma criança no período de aquisição de linguagem. Essa gramática difere da gramática de um indivíduo adulto no que diz respeito a sua maturação. Além das crianças em fase de aquisição de linguagem, é considerado ainda que indivíduos neurolesionados também podem apresentar uma gramática desviante. Tais desvios concernem não a aspectos maturacionais, mas sim a impedimentos provocados pela lesão cerebral e que podem estar revelados na expressão linguística desses indivíduos. Assim sendo, podemos dizer que determinadas lesões cerebrais, incluindo os prejuízos provocados pelas síndromes demenciais, podem levar a alterações na expressão linguística. Neste trabalho, pretende-se contribuir para o entendimento da representação mental da linguagem a partir da avaliação da expressão linguística de indivíduos que apresentam uma gramática desviante. Mais especificamente, pretende-se investigar a linguagem de indivíduos com a demência do tipo Alzheimer (ou DTA).

Diversos estudiosos voltados para a avaliação de indivíduos que apresentam uma gramática desviante estão inclinados a investigar alterações linguísticas na afasia de Broca, uma patologia de linguagem caracterizada por gerar um déficit exclusivamente linguístico, sem alterar outros sistemas cognitivos. Além da contribuição oferecida pelos estudos sobre afasia, é possível também contribuir para uma teoria linguística que explique como a linguagem está representada na mente a partir da investigação de doenças neurológicas progressivas provenientes de síndromes demenciais, como é o caso da DTA.

Considerando que a linguagem tem sido alvo de investigação quando se trata da DTA e que a sua deterioração é consensualmente assumida (cf. MANSUR et al., 2005, p. 300), existem muitos estudos que, para alcançar, de alguma maneira, o entendimento da linguagem e da sua relação com outros sistemas cognitivos, se voltam para a investigação de patologias como a DTA. Observa-se que esses estudos buscam descrever os sintomas relacionados aos problemas de linguagem na DTA, bem como esclarecer questões relativas aos comprometimentos cognitivos subjacentes a esses sintomas. Uma vez que múltiplos impedimentos cognitivos acometem os pacientes portadores dessa demência, é possível que as eventuais alterações encontradas na expressão linguística sejam decorrentes ou de um comprometimento em sistemas cognitivos não linguísticos, como o sistema conceptual, por exemplo, ou de um comprometimento especificamente no sistema da linguagem propriamente dito.

Em consonância com os estudos que buscam descrever as alterações linguísticas e esclarecer questões relativas aos comprometimentos cognitivos subjacentes a essas alterações na DTA, optamos neste trabalho por avaliar a linguagem nessa demência no que concerne a dois fenômenos específicos, a saber: tempo e aspecto. Tais fenômenos correspondem a traços linguísticos conceptualmente motivados, o que nos faz pensar que tanto um comprometimento em sistemas cognitivos não linguísticos como um comprometimento no sistema da linguagem pode levar a possíveis alterações na expressão desses traços.

Assim sendo, objetiva-se com este trabalho investigar a expressão linguística de tempo e aspecto de prováveis pacientes portadores da DTA, a fim de contribuir para a elucidação do questionamento acerca da natureza das alterações reveladas na expressão linguística desses pacientes. Para tanto, foi realizada uma investigação longitudinal tanto do sistema conceptual quanto da expressão linguística de tempo e aspecto de um provável paciente portador da demência em questão.

Este trabalho está dividido em 5 seções: na primeira seção, encontra-se a fundamentação teórica deste trabalho, na qual serão apresentados os problemas linguísticos na DTA comumente descritos na literatura; na segunda seção, encontra-se a metodologia adotada, na qual serão descritos os testes utilizados a fim de alcançar os objetivos propostos; na terceira seção, encontram-se os resultados dos testes aplicados; por fim, na quarta seção, encontram-se a discussão e a análise dos resultados obtidos.

\section{Fundamentação teórica}

$\mathrm{O}$ estudo da linguagem pode ser realizado a partir de diferentes perspectivas teóricas. Um dos paradigmas da Linguística que tem se preocupado com a caracterização do sistema linguístico e da sua interação com os demais sistemas cognitivos da mente humana tem sido o Gerativismo. De acordo com esse paradigma, o conhecimento de linguagem corresponde a um sistema específico, que é, em certo grau, autônomo em relação aos demais sistemas que compõem a mente. Isso significa dizer que a representação dessa gramática na mente é modular (cf. CHOMSKY, 1986, p. 12-13).

Baseando-se nessa teoria, considera-se objeto de investigação o estudo de determinados fenômenos linguísticos. Os fenômenos linguísticos que se busca investigar são conhecidos na teoria como traços ${ }^{2}$. Uma

\footnotetext{
2 Neste contexto, os traços a que se faz referência são os traços sintáticos.
} 
vez que se adote uma teoria baseada em traços, surgem algumas perguntas centrais para a pesquisa linguística. Uma delas diz respeito aos traços que compõem o conhecimento linguístico de um falante.

O estudo dos traços, de acordo com os desdobramentos dessa teoria sugeridos por Chomsky (1995) no Programa Minimalista, está baseado em pressupostos como o da interpretabilidade, por exemplo. Essa noção é fundamental para determinar os traços formais que são relevantes para a faculdade da linguagem, pois, segundo esse pressuposto, somente projetam nódulos na árvore sintática traços que sejam interpretáveis nos sistemas de desempenho. Sendo assim, para que um traço projete nódulo na sintaxe, é necessário que o mesmo seja interpretável pelo sistema articulatório-perceptual, de um lado, e pelo sistema conceptual, de outro.

Dentre os traços flexionais (e, portanto, funcionais) interpretáveis conceptualmente que, dessa forma, ganham destaque nos estudos gerativistas voltados para a sintaxe, podem ser mencionados os de tempo e de aspecto. Por um lado, tempo é uma noção dêitica que localiza os eventos em relação a um ponto de referência e, por outro lado, aspecto é uma noção não dêitica que está relacionada às diferentes formas de se observar a constituição temporal interna de uma determinada situação (cf. COMRIE, 1976, p. 3). Tendo em vista as informações semânticas referentes a tempo e a aspecto, pode ser dito que ambos parecem se tratar de traços flexionais interpretáveis semanticamente e, portanto, legíveis no sistema conceptual.

Neste trabalho, pretende-se investigar eventuais alterações de linguagem na DTA, mais especificamente alterações relativas à expressão linguística de tempo e aspecto. Pretende-se, sobretudo, avaliar o(s) comprometimento(s) cognitivo(s) subjacente(s) a essas eventuais alterações. Assim sendo, faz-se necessário detalhar alguns dos problemas de linguagem comumente descritos na literatura que acometem esses pacientes, bem como os distúrbios cognitivos aos quais tais problemas são atribuídos. Tal detalhamento apresenta-se nos parágrafos a seguir.

As síndromes demenciais de ordem degenerativa são caracterizadas pelo comprometimento progressivo das funções cognitivas. Em relação especificamente à DTA, funções cognitivas como a memória encontram-se prejudicadas, podendo tal prejuízo se estender a outros sistemas, como ao sistema dos conceitos e ao sistema da linguagem, por exemplo. O comprometimento das funções cognitivas na DTA levou à caracterização de um quadro clínico que prevê sintomas como desorientação, confusão, perdas da capacidade de julgamento, mudanças de comportamento. Além dos sintomas mencionados, assume-se que alterações reveladas na linguagem constituem um dos problemas provocados pela DTA.
Dentre as descrições que têm sido propostas acerca das alterações reveladas na expressão linguística de pacientes portadores da DTA, pode ser citado o trabalho de Huff (1988) e, mais recentemente, o de Mansur et al. (2005). Os autores acreditam que o processo de deterioração da linguagem percorre três estágios. No decorrer desse processo, são acometidas tanto as habilidades receptivas - de compreensão - quanto as habilidades expressivas - de produção -. Podem estar comprometidos ainda aspectos pragmáticos, semânticos, lexicais, sintáticos e fonológicos nessa demência.

Esses autores admitem que a progressão de algumas perdas na DTA parece seguir um percurso esperado. Em relação especificamente à linguagem, no primeiro estágio de prevalência da demência, observa-se, em geral, uma relativa preservação da fluência, apesar da grande dificuldade de nomeação. O discurso é marcado por circunlóquios, apresentando diversas pausas em decorrência da tentativa de buscar as palavras que se pretende empregar na fala. No segundo estágio, as parafasias fonêmicas e semânticas são características marcantes. Além disso, as expressões verbais parecem ficar cada vez mais reduzidas, o que reduz a complexidade sintática das orações que produzem. Por fim, no último estágio, o discurso se restringe a repetições sem sentido de palavras e a produção de sons sem sentido. Alguns pacientes ficam mudos nessa fase.

Dentre os estudos cujo objetivo era descrever e explicar os problemas de linguagem na DTA, muitos tinham como objeto de investigação as alterações de ordem lexical e semântica. Conforme relatado por Kempler et al. (1999, p. 228), os problemas de nomeação de pacientes com a DTA provenientes de problemas léxico-semânticos foram exaustivamente descritos e analisados em termos de frequência e da natureza do comprometimento cognitivo subjacente. Além disso, segundo Mansur et al. (2005, p. 300), parece haver um consenso no que diz respeito à prevalência dessas alterações e o seu processo de evolução.

Pode-se dizer, dessa forma, que a deterioração das habilidades léxico-semânticas foi cuidadosamente apurada, visto que se trata de um dos sintomas linguísticos mais facilmente percebidos. Além de problemas léxicosemânticos, alguns dos problemas sintáticos provocados pela DTA também são relatados na literatura. No entanto, não parece haver um consenso a respeito da natureza desses problemas. Para exemplificar a falta de acordo entre os autores que estudam a sintaxe de pacientes com DTA, podem ser destacados os estudos de Rochon, Waters e Caplan (1994) e de Grober e Bang (1995).

Rochon, Waters e Caplan (1994) promoveram um estudo sobre a compreensão de sentenças na DTA. Os autores buscavam investigar, através da aplicação de um 
teste de relacionamento imagem-sentença, o desempenho de pacientes com essa demência, em comparação com o desempenho de indivíduos controles, ao lidar com sentenças que apresentavam diferentes graus de complexidade sintática. A análise dos resultados proposta pelos autores sugere (1) que as alterações encontradas não estavam correlacionadas à complexidade das sentenças e (2) que os pacientes apresentaram problemas com sentenças que tinham duas proposições, ou seja, com sentenças que continham dois verbos. Esse desempenho insatisfatório foi explicado pelos autores como decorrente de um problema pós-interpretativo, uma vez que sentenças com duas proposições demandam uma carga processual maior durante a realização da tarefa. Sendo assim, pode-se concluir que, de acordo com os dados obtidos por Rochon, Waters e Caplan (1994), a natureza das alterações de compreensão de sentenças de pacientes com a DTA é decorrente de um comprometimento em sistemas cognitivos não linguísticos, estando, nesse caso, a sintaxe preservada.

O estudo de Grober e Bang (1995) estava baseado em uma metodologia a partir da qual também era prevista a utilização de um teste de relacionamento imagem-sentença. Através de tal experimento, buscavase investigar a compreensão dos pacientes portadores da DTA ao lidar com sentenças que fossem semanticamente reversíveis e com sentenças que fossem semanticamente irreversíveis. Tanto as sentenças reversíveis quanto as sentenças irreversíveis poderiam estar ora na voz ativa ora na voz passiva. Foi concluído pelos autores, com base nos resultados obtidos, que as dificuldades de compreensão de sentenças de pacientes portadores da DTA eram decorrentes de um comprometimento genuinamente sintático, visto que os pacientes investigados apresentaram dificuldades em compreender sentenças cuja interpretação dependia necessariamente da análise sintática, como era o caso das sentenças semanticamente reversíveis, por exemplo.

Recorrendo a estudos mais recentes que buscam investigar eventuais alterações reveladas na expressão linguística de pacientes portadores da DTA e que tratam especificamente de possíveis alterações flexionais, podemos mencionar dois estudos: o estudo de Kavé e Levy (2003), em que se defende a preservação da expressão linguística e o estudo de Martins (2010), em que se advoga em favor de uma deterioração do sistema linguístico, cujos danos estão refletidos na expressão linguística desses pacientes. A seguir, serão apresentados a metodologia e os resultados a que chegaram esses autores.

Kavé e Levy (2003), com o objetivo de investigar a existência de possíveis alterações na expressão linguística de pacientes com a DTA, investigaram as categorias de tempo e concordância em pacientes falantes de hebraico.
A metodologia desenvolvida foi dividida em duas partes: a primeira se tratava de uma tarefa on-line de leitura automonitorada e a segunda se tratava de uma tarefa off-line de julgamento de agramaticalidade. Com base nos dados obtidos por meio de ambos os experimentos, os autores em questão concluíram que o desempenho de pacientes com DTA é semelhante ao desempenho de indivíduos normais em tarefas que requerem o processamento da flexão, uma vez que os pacientes alcançaram resultados semelhantes aos dos indivíduos controles, mesmo sendo rico o paradigma flexional do hebraico. Com base nesses resultados, pôde ser dito que, no que diz respeito aos fenômenos investigados, o sistema linguístico dos pacientes com a DTA estava preservado, mantendo-se inalterada a expressão linguística da flexão.

Se Rochon, Waters e Caplan (1994) e Kavé e Levy (2003) parecem encontrar resultados que advogam em favor da proposta de que o sistema linguístico está preservado em pacientes com a DTA, o mesmo não ocorre com Martins (2010). Pretendia-se investigar, assim como Kavé e Levy (2003), a flexão, mais especificamente as categorias funcionais de tempo e aspecto, em pacientes com a DTA falantes do português do Brasil. A autora utilizou uma metodologia que se baseava na aplicação de um teste de julgamento de agramaticalidade, bem como na aplicação de um teste de produção. Tendo em vista que um dos objetivos da autora era investigar a origem de possíveis alterações na expressão linguística de tempo e aspecto desses pacientes, foi proposta uma comparação dos dados linguísticos de pacientes com déficit cognitivo com dados de pacientes sem déficit cognitivo, sendo a presença ou ausência de tal déficit avaliada através da aplicação de um teste neuropsicológico.

A análise dos resultados de ambos os testes sugere que, em comparação aos indivíduos controles, os pacientes portadores da DTA com déficit cognitivo e sem esse déficit que foram submetidos à avaliação proposta apresentaram problemas na expressão linguística. Esses problemas foram atribuídos a um comprometimento essencialmente linguístico, uma vez que pacientes cognitivamente preservados apresentaram problemas na expressão linguística, o que pode ser um indicativo de uma desintegração do sistema linguístico propriamente dito. Contudo, verificou-se ainda um desempenho mais agravado dos pacientes com déficit cognitivo em comparação com os dados dos pacientes sem déficit cognitivo. A autora propôs uma interpretação segundo a qual as alterações na expressão linguística dos pacientes investigados são decorrentes de um comprometimento do sistema linguístico, seguindo a mesma direção de Grober e Bang (1995), podendo o desempenho linguístico ser ainda mais afetado devido a comprometimentos em outros sistemas mentais que interagem com a linguagem. 
Conforme ilustrado por meio dos estudos apresentados anteriormente, pode-se dizer que a DTA é uma patologia de linguagem capaz de provocar alterações relativas à expressão linguística de fenômenos sintáticos bem como de fenômenos léxico-semânticos. No entanto, no tocante à expressão linguística de fenômenos flexionais, não parece haver um consenso quanto à sua deterioração. Essa falta de consenso é exemplificada por meio da divergência das análises propostas nos estudos de Kavé e Levy (2003), de um lado, e de Martins (2010), de outro.

Uma vez que se observa um desacordo quanto à eventual existência de problemas flexionais na DTA, faz-se necessária a proposição de novas investigações que auxiliem a elucidação dessa questão. Assim sendo, objetiva-se investigar possíveis alterações flexionais nessa demência. Mais especificamente, objetiva-se investigar a expressão linguística de tempo e de aspecto de pacientes portadores da DTA.

Considerando que tempo e aspecto são traços linguísticos interpretáveis conceptualmente, é possível que um comprometimento no sistema linguístico ou um comprometimento no sistema conceptual explique as eventuais alterações encontradas na expressão linguística. Desse modo, objetiva-se, ainda, contribuir para o entendimento acerca do comprometimento cognitivo que se encontra subjacente a possíveis alterações na expressão linguística de tempo e aspecto, tendo em vista o quadro clínico de múltiplos impedimentos cognitivos característico da DTA.

A seguir, apresentamos a metodologia utilizada a fim de alcançar os objetivos propostos.

\section{Metodologia}

\subsection{Tipo de estudo}

A fim de alcançar os objetivos propostos, foi desenvolvido um estudo que pode ser caracterizado, de um lado, como estudo de caso e, de outro, como estudo longitudinal.

Optou-se por desenvolver um estudo de caso com base em trabalhos como o de Nespoli et al. (2010), por exemplo, em que se observou uma grande variabilidade no que diz respeito ao desempenho linguístico de pacientes com DTA. Evidenciou-se, assim, a relevância de se analisar individualmente o desempenho de pacientes acometidos por essa demência.

Além de a presente pesquisa ser caracterizada por se tratar de estudo de caso, optou-se também por realizar um estudo longitudinal. Esse tipo de estudo se define por serem coletados periodicamente os dados de um mesmo informante. Estudos longitudinais que buscam avaliar as funções cognitivas de pacientes com DTA são importantes no sentido de melhor promoverem a compreensão do processo cognitivo e biológico natural da demência, oferecendo, desse modo, uma descrição acurada das perdas. Dessa forma, é possível que, em algum momento da investigação, possam ser detectadas alterações relativas a tempo e aspecto.

\subsection{0 informante}

Com o objetivo de desenvolver este estudo, foi selecionado um paciente do sexo feminino provável portador da DTA com sessenta e sete anos. O seu diagnóstico havia sido dado há aproximadamente quatro anos antes do início da pesquisa. O paciente tinha Ensino Superior na área de Engenharia e apresentava, na época da investigação, uma vida social bastante ativa e frequentava grupos de estudos. Essa intensa socialização parece ser uma característica bastante relevante da vida do paciente, pois permitiu que ele colaborasse ativamente com o estudo.

\subsection{Os testes}

O paciente selecionado foi submetido a aplicações de três testes: a dois testes neuropsicológicos, que permitem uma avaliação neuropsicológica mais geral e conceptual, e a um teste linguístico, que permite uma avaliação da expressão linguística de tempo e aspecto. Cada teste foi aplicado ao paciente quatro vezes em um intervalo de seis meses entre cada aplicação. Os dados da primeira aplicação de cada teste foram tomados como parâmetro para a detecção de possíveis perdas cognitivas linguísticas e não linguísticas que pudessem emergir posteriormente. Desse modo, optamos por chamar a primeira aplicação de aplicação controle 3 .

O primeiro teste neuropsicológico consistia no MiniExame do Estado Mental (MEEM), uma versão brasileira proposta por Caramelli e Nitrini (2000) do Mini-Mental State Examination. Pretendia-se, com a aplicação desse teste, avaliar possíveis perdas cognitivas ao longo do tempo e o grau dessas perdas. Esse teste é composto por trinta perguntas ou comandos, devendo ser contabilizado um ponto para cada acerto na sua realização. Foram utilizados os parâmetros de Folstein, Folstein e Fanjiang (2000), autores do Mini-Mental State Examination, para avaliar os diferentes graus de impedimentos cognitivos. Os autores estabelecem que notas entre vinte e um e vinte e seis podem indicar um impedimento cognitivo leve; notas entre onze e vinte, um impedimento cognitivo moderado; notas entre zero e dez, um impedimento cognitivo severo.

\footnotetext{
Utilizamos a primeira aplicação como parâmetro de análise em detrimento da utilização de dados de indivíduos controles. Isso justifica o fato de a primeira aplicação ter sido nomeada de aplicação controle.
} 
O segundo teste neuropsicológico consistia em um teste de sequência lógica, que foi elaborado especialmente para este estudo, com o objetivo de detectar alterações conceptuais que pudessem estar relacionadas ao conceito de tempo e que não poderiam ser capturadas pelo MEEM. Sendo assim, foi avaliada não só a cognição mais geral do paciente através da utilização do MEEM mas também o conhecimento lógico e temporal através da utilização do teste de sequência lógica. Esse teste é composto por dez sequências, sendo cada sequência composta por quatro imagens que, ao ser ordenada apropriadamente, representa uma situação específica. As imagens utilizadas se referem a fotografias que ilustram o mesmo personagem realizando atividades em todas as sequências. Para que fosse minimizada a possibilidade de se obter respostas subjetivas na ordenação das sequências, a primeira imagem de cada sequência era dada pelo pesquisador e era informado que se tratava da primeira imagem da sequência. Dessa maneira, ao informante, era apresentada uma sequência de cada vez com as imagens em ordem aleatória, exceto a primeira. As três imagens de cada sequência deveriam ser organizadas em ordem de acontecimento, tanto do ponto de vista lógico, através da relação de causa e efeito, quanto do ponto de vista temporal, através da relação de sucessão, de modo que fosse conferida à sequência uma ordenação que expressasse uma possibilidade real de concretização da situação.

O teste linguístico, que também foi elaborado especialmente para este estudo, consistia em um teste de produção de preenchimento de lacuna com o objetivo de detectar alterações na expressão linguística de tempo e de aspecto. Esse teste é composto por quarenta sentenças, sendo que metade das sentenças é composta por sentenças-alvo e a outra metade é composta por sentenças distratoras. As sentenças apresentam uma lacuna a ser completada por uma das três opções de resposta disponíveis.

No que diz respeito às lacunas das sentenças que são alvo de investigação, esperava-se que elas fossem preenchidas com um verbo/locução verbal com traços temporais e aspectuais compatíveis com a expressão adverbial da sentença. Isto é, a expressão adverbial de cada sentença era o indicador do verbo que deveria ser selecionado de modo que sua morfologia e aspecto lexical deveriam apresentar compatibilidade com os traços sintáticos temporais e aspectuais da expressão adverbial. $\mathrm{O}$ verbo da primeira opção das sentenças-alvo apresenta uma morfologia de tempo passado e de aspecto perfectivo, o verbo da segunda opção apresenta uma morfologia de tempo passado e de aspecto imperfectivo, podendo ser ora durativa ora habitual, e o verbo da terceira opção apresenta morfologia de tempo presente e de aspecto imperfectivo, podendo ser ora durativa ora habitual.
Além de vinte sentenças-alvo, foram utilizadas vinte sentenças distratoras, cujo objetivo era distrair a atenção do informante quanto ao objeto de análise, ou seja, quanto às informações temporais e aspectuais presentes na sentença. A lacuna dessas sentenças diz respeito à preposição que deve completar a sentença e que seja compatível com o verbo da sentença.

Em suma, pode-se dizer que, de um lado, buscamos avaliar possíveis declínios cognitivos mais gerais e conceptuais e, de outro lado, buscamos avaliar possíveis alterações na expressão linguística de tempo e aspecto. A comparação dos resultados obtidos por meio da aplicação dos testes neuropsicológicos com os resultados obtidos por meio da aplicação do teste linguístico permite a investigação da natureza das possíveis alterações de expressão linguística encontradas.

\section{Resultados}

\subsection{Resultados da aplicação controle}

Conforme dito na seção anterior, os dados obtidos na primeira aplicação dos testes foram tomados como parâmetro para a verificação de possíveis perdas que pudessem ser reveladas por meio das aplicações posteriores dos testes. Considerando a aplicação controle dos testes neuropsicológicos, deve ser mencionado que o paciente obteve uma nota de vinte e três pontos de trinta possíveis no MEEM, o que caracteriza um comprometimento cognitivo leve. Além disso, ordenou adequadamente nove sequências de dez que compunham o teste de sequência lógica. Observou-se, assim, uma relativa preservação neuropsicológica ${ }^{4}$ na aplicação controle.

Em relação ao resultado da aplicação controle do teste de preenchimento de lacuna, deve ser dito que o paciente preencheu dezenove lacunas com as formais verbais compatíveis em tempo e aspecto com a expressão adverbial da sentença de vinte sentenças-alvo possíveis. Observou-se, assim, uma preservação da expressão linguística de tempo e aspecto na aplicação controle. $\mathrm{Na}$ Tabela 1 a seguir, podem ser observados os resultados da aplicação controle.

Tabela 1. Resultado do aproveitamento do paciente nos testes na aplicação controle.

\begin{tabular}{lc}
\hline \multicolumn{1}{c}{ Aplicação } & Controle \\
\hline MEEM - grau de impedimento & 23 - leve \\
Teste de sequência lógica & 9 sequências \\
Teste de preenchimento de lacuna* & 19 \\
\hline
\end{tabular}

* Os resultados do teste de preenchimento de lacuna apresentados na Tabela 1 se referem ao desempenho do paciente ao lidar com as sentenças-alvo desse teste.

4 Considera-se relativa a preservação neuropsicológica, uma vez que o pacien-
te apresentou um comprometimento apontado pelo MEEM, ainda que leve. 


\subsection{Resultados da segunda aplicação}

Considerando a segunda aplicação dos testes neuropsicológicos, deve ser mencionado que o paciente obteve uma nota de dezesseis pontos de trinta possíveis no MEEM, o que caracteriza um comprometimento cognitivo moderado. Além disso, ordenou adequadamente sete sequências de dez que compunham o teste de sequência lógica. Observou-se, assim, um declínio neuropsicológico em relação aos dados obtidos por meio da aplicação controle.

Em relação ao resultado da segunda aplicação do teste de preenchimento de lacuna, deve ser dito que o paciente preencheu dezessete lacunas com as formais verbais compatíveis em tempo e aspecto com a expressão adverbial da sentença de vinte sentenças-alvo possíveis. Observou-se, assim, uma alteração na expressão linguística de tempo e aspecto em relação aos dados obtidos por meio da aplicação controle. Na Tabela 2 a seguir, podem ser observados os resultados da segunda aplicação em comparação com os resultados da aplicação controle.

Tabela 2. Resultado do aproveitamento do paciente nos testes na segunda aplicação em relação à aplicação controle.

\begin{tabular}{lcc}
\hline \multicolumn{1}{c}{ Aplicação } & Controle & Segunda \\
\hline MEEM - grau de impedimento & 23 - leve & 16 - moderado \\
Teste de sequência lógica & 9 sequências & 7 sequências \\
Teste de preenchimento de lacuna* & 19 & 17 \\
\hline
\end{tabular}

* Os resultados do teste de preenchimento de lacuna apresentados na Tabela 2 se referem ao desempenho do paciente ao lidar com as sentenças-alvo desse teste.

\subsection{Resultados da terceira aplicação}

Considerando a terceira aplicação dos testes neuropsicológicos, deve ser mencionado que o paciente obteve uma nota de treze pontos de trinta possíveis no MEEM, o que caracteriza um comprometimento cognitivo moderado. Além disso, ordenou adequadamente seis sequências de dez que compunham o teste de sequência lógica. Observou-se, assim, um declínio neuropsicológico em relação aos dados obtidos por meio da aplicação controle e por meio da segunda aplicação.

Em relação ao resultado da terceira aplicação do teste de preenchimento de lacuna, deve ser dito que o paciente preencheu quinze lacunas com as formais verbais compatíveis em tempo e aspecto com a expressão adverbial da sentença de vinte sentenças-alvo possíveis. Observou-se, assim, uma alteração ainda maior na expressão linguística de tempo e aspecto em relação aos dados obtidos por meio da aplicação controle e da segunda aplicação. Na Tabela 3 a seguir, podem ser observados os resultados da terceira aplicação em comparação com os resultados da aplicação controle e da segunda aplicação.

Tabela 3. Resultado do aproveitamento do paciente nos testes na terceira aplicação em relação à aplicação controle e à segunda aplicação.

\begin{tabular}{lccc}
\hline \multicolumn{1}{c}{ Aplicação } & Controle & Segunda & Terceira \\
\hline $\begin{array}{l}\text { MEEM - grau de } \\
\text { impedimento }\end{array}$ & 23 - leve & 16 - moderado & 13 - moderado \\
$\begin{array}{l}\text { Teste de sequência } \\
\text { lógica }\end{array}$ & 9 sequências & 7 sequências & 6 sequências \\
$\begin{array}{l}\text { Teste de preenchimento } \\
\text { de lacuna* }\end{array}$ & 19 & 17 & 15 \\
\hline * Os resultados do teste de preenchimento de lacuna apresentados na Tabela 3 se \\
referem ao desempenho do paciente ao lidar com as sentenças-alvo desse teste.
\end{tabular}

\subsection{Resultados da quarta aplicação}

Considerando a quarta aplicação dos testes neuropsicológicos, deve ser mencionado que o paciente obteve uma nota de quinze pontos de trinta possíveis no MEEM, o que caracteriza um comprometimento cognitivo moderado. Além disso, ordenou adequadamente seis sequências de dez que compunham o teste de sequência lógica. Observou-se, assim, um declínio neuropsicológico em relação aos dados obtidos por meio da aplicação controle e por meio da segunda aplicação. No entanto, observou-se uma estabilidade neuropsicológica em relação aos dados obtidos por meio da terceira aplicação.

Em relação ao resultado da quarta aplicação do teste de preenchimento de lacuna, deve ser dito que o paciente preencheu nove lacunas com as formais verbais compatíveis em tempo e aspecto com a expressão adverbial da sentença de vinte sentenças-alvo possíveis. Observou-se, assim, uma alteração ainda maior na expressão linguística de tempo e aspecto em relação aos dados obtidos por meio da aplicação controle, da segunda aplicação e da terceira aplicação. Na Tabela 4 abaixo, podem ser observados os resultados da quarta aplicação em comparação com os resultados da aplicação controle, da segunda aplicação e da terceira aplicação.

Tabela 4. Resultado do aproveitamento do paciente nos testes na quarta aplicação em relação à aplicação controle, à segunda aplicação e à terceira aplicação.

\begin{tabular}{lcccc}
\hline \multicolumn{1}{c}{ Aplicação } & Controle & Segunda & Terceira & Quarta \\
\hline MEEM - grau de impedimento & $23-$ leve & 16 - moderado & $13-$ moderado & $15-$ moderado \\
Teste de sequência lógica & 9 sequências & 7 sequências & 6 sequências & 6 sequências \\
Teste de preenchimento de lacuna* & 19 & 17 & 15 & 9 \\
\hline
\end{tabular}

* Os resultados do teste de preenchimento de lacuna apresentados na Tabela 4 se referem ao desempenho do paciente ao lidar com as sentenças-alvo desse teste. 
Em resumo, pode ser dito que, através dos resultados obtidos neste estudo, detectou-se um declínio até a terceira aplicação e uma estabilidade na quarta aplicação dos testes neuropsicológicos e um comprometimento cada vez maior na expressão linguística de tempo e aspecto até a quarta aplicação do teste de preenchimento de lacuna.

\section{Discussão}

A análise dos resultados da avaliação neuropsicológica indicou a existência de alterações de natureza cognitiva mais geral e conceptual na DTA por meio do MEEM e por meio do teste de sequência lógica, respectivamente. Em paralelo, a análise dos resultados do teste de preenchimento de lacuna indicou a existência de alterações na expressão linguística de tempo e aspecto na DTA.

Do ponto de vista longitudinal, pode ser dito que parte dessas alterações aumentou ao longo tempo. Em relação à cognição mais geral e aos conceitos, observouse uma deterioração que pôde ser detectada até a terceira aplicação dos testes, apresentando uma estabilidade na quarta aplicação em relação à terceira aplicação. Em relação à expressão linguística tanto de tempo quanto de aspecto, a deterioração observada se estendeu até a quarta aplicação. Os resultados de natureza neuropsicológica e os resultados de natureza linguística de cada aplicação foram comparados.

Ao confrontar os dados de natureza neuropsicológica aos dados de natureza linguística, conforme pode ser verificado na Tabela 4 apresentada anteriormente, observa-se uma correlação entre os declínios cognitivos mais gerais e conceptuais e o comprometimento cada vez maior na expressão linguística de tempo e aspecto até a terceira aplicação dos testes. Na quarta aplicação, as alterações de ordem cognitiva mais geral e conceptual estão estabilizadas ao passo que as alterações linguísticas de tempo e aspecto seguem o mesmo curso de declínio.

Quanto aos objetivos deste trabalho, buscou-se contribuir para a elucidação do questionamento acerca da origem de eventuais alterações na expressão linguística de tempo e aspecto na DTA. Uma vez que foram detectadas tais alterações, faz-se necessário aludir aos comprometimentos cognitivos subjacentes. A DTA é primordialmente conhecida pelo seu caráter degenerativo que leva a um comprometimento progressivo das funções cognitivas. Sendo assim, é muito possível que, em algum estágio da doença, o sistema linguístico seja afetado. A questão que se coloca neste estudo é se os problemas de expressão linguística de tempo e aspecto, detectados por meio das aplicações do teste de preenchimento de lacuna, são oriundos de um comprometimento que atinge esse sistema ou sistemas não linguísticos.
Com base nos dados obtidos até a terceira aplicação dos testes, parece que a expressão linguística de tempo e aspecto pode ser alterada em razão de um comprometimento cognitivo mais geral e conceptual, mas também pode ser alterada em função de um comprometimento no sistema da linguagem. Isso significa dizer que, embora haja uma correlação entre os declínios cognitivos mais gerais e conceptuais e o comprometimento cada vez maior na expressão linguística de tempo e aspecto até a terceira aplicação dos testes, não é possível afirmar somente com base nesses dados se a origem diz respeito a um comprometimento na linguagem ou em outros sistemas cognitivos não linguísticos. É possível que haja também um declínio no sistema da linguagem que acompanha o declínio cognitivo mais geral e que acarreta o problema na expressão linguística.

Já o comprometimento ainda maior na expressão linguística de tempo e aspecto verificado através dos resultados da quarta aplicação do teste de preenchimento de lacuna não está correlacionado a um declínio cognitivo mais geral e conceptual, visto que foi observada uma estabilidade neuropsicológica da terceira para a quarta aplicação dos testes. Uma possível explicação para os resultados da quarta aplicação do teste linguístico pode ir ao encontro das conclusões a que chegaram autores como Grober e Bang (1995) e Martins (2010). Sendo assim, é possível que um distúrbio no sistema linguístico seja o responsável por ter agravado ainda mais os problemas na expressão linguística de tempo e aspecto detectados por meio da quarta aplicação dos testes.

Tendo em vista a interpretação proposta para os resultados encontrados, podemos concluir que alcançamos os objetivos estabelecidos para este trabalho. Primeiramente, pretendíamos investigar possíveis alterações na expressão linguística de tempo e de aspecto de pacientes portadores da DTA. Em relação a esse objetivo, podemos dizer que a expressão linguística de tempo e aspecto pode estar alterada em algum estágio da DTA. Pretendíamos ainda contribuir para o entendimento acerca do comprometimento cognitivo que se encontra subjacente a essas possíveis alterações. Em relação a esse objetivo, podemos dizer que ao menos parte das alterações de expressão linguística encontradas parece decorrer de um comprometimento no sistema da linguagem propriamente dito.

Por fim, um aspecto que merece ser mencionado diz respeito a uma questão teórica sobre representação mental da linguagem. Assumindo que possa haver um comprometimento cognitivo que incide especificamente no sistema linguístico na DTA que parece não progredir paralelamente às perdas cognitivas mais gerais e conceptuais, pode ser dito que há uma perda seletiva das funções cognitivas. Assim sendo, é possível propor, com base nos dados obtidos, uma dissociação entre a função 
cognitiva conceptual e a função cognitiva linguística. Essa dissociação encontrada pode constituir mais uma evidência empírica em favor da proposta de modularidade da mente, pressuposto adotado neste trabalho.

\section{Considerações finais}

A investigação proposta para este estudo permitiu que fossem detectadas longitudinalmente o surgimento de alterações reveladas na expressão linguística de tempo e aspecto na DTA. Verificou-se que parte dessas alterações parece decorrer de um comprometimento genuinamente no sistema da linguagem, uma vez que se detectou o surgimento de alterações linguísticas em meio à estabilidade neuropsicológica. Sendo assim, pode-se concluir que um comprometimento do sistema linguístico pode ser um dos prejuízos responsáveis pelas alterações linguísticas na DTA.

Um possível desdobramento deste estudo poderá contemplar, dentre outras, as seguintes questões: (1) o aprimoramento dos testes elaborados com o objetivo de melhor capturar as alterações de tempo e aspecto, inclusive um possível comprometimento seletivo entre essas categorias; (2) a coleta de dados de um grupo de pacientes portadores da DTA, com mesmo tempo de demência, a fim de contribuir para a discussão a respeito da existência ou não de certos padrões relacionados à progressão das alterações linguísticas na DTA.

\section{Referências}

CARAMELLI, Paulo; NITRINI, Ricardo. Como avaliar de forma breve e objetiva o estado mental de um paciente? Rev. Assoc. Med. Bras, v. 46, n. 4, p. 301-304, 2000.

CHOMSKY, Noam. Knowledge of language: its nature, origin and use. New York: Praeger, 1986.

CHOMSKY, Noam. The Minimalist Program. Cambridge, MA: MIT Press, 1995.

COMRIE, Bernard. Aspect. Cambridge: Cambridge University Press, 1976.
FOLSTEIN, Marshal F.; FOLSTEIN, Susan E.; FANJIANG, Gary. Mini-Mental State Examination: Clinical Guide. Lutz, FL: Psychological Assessment Resources, Inc., 2000.

GROBER, Ellen; BANG, Shereen. Sentence comprehension in Alzheimer's disease. Developmental Neuropsychology, v. 11, p. $95-107,1995$.

HUFF, Jacob F. The disorder of naming in Alzheimer's disease. In: LIGHT, Leah L.; BURKE, Deborah M. (Org.). Language, memory, and aging. Cambridge: Cambridge University Press, 1988. p. 209-220.

KAVÉ, Gitit; LEVY, Yonata. Sensitivity to gender, person, and tense inflection by persons with Alzheimer's disease. Brain and Language, v. 87, p. 267-277, abr. 2003.

KEMPLER, Daniel.; ALMOR, Amit; MACDONALD, Maryellen C.; ANDERSEN, Elaine S. Working with limited memory: Sentence comprehension in Alzheimer's disease. In: KEMPER, Susan.; KLIEGL, Reinhold (Org.). Constraints on Language: Aging, Grammar, and Memory. Boston, US: Kluwer Academic Publishers, 1999. p. 227-247.

MANSUR, Letícia Lessa; CARTHERY, Maria Teresa; CARAMELLI, Paulo; NITRINI, Ricardo. Linguagem e cognição na Doença de Alzheimer. Psicol. Reflex. Crit., Porto Alegre, v. 18, n. 3, p. 300-307, dez. 2005.

MARTINS, Adriana Leitão. A desintegração do tempo na demencia do tipo Alzheimer. 2010. 239 fls. Tese (Doutorado em Letras) - Universidade Federal do Rio de Janeiro, Rio de Janeiro, 2010

NESPOLI, Juliana Barros; NOVAES, Celso Vieira; RODRIGUES, Fernanda de Carvalho; MARTINS, Adriana Leitão. Variability of the linguistic performance of patients with probable dementia of the Alzheimer type. In: FRANÇA, Aniela Improta; MAIA, Marcus (Org.). Papers in Psycholinguistics. Rio de Janeiro: Imprinta, 2010. p. 356-361.

ROCHON, Elizabeth; WATERS, Gloria S.; CAPLAN, David. Sentence comprehension in patients with Alzheimer's disease. Brain and Language, v. 46, p. 332-349, 1994.

Recebido: 03 de fevereiro de 2016

Aprovado: 02 de junho de 2016

Contato: juliananespoli@yahoo.com.br cnovaes@ufrj.br 